\title{
El liderazgo servidor y la satisfacción laboral en estudiantes de maestría en administración de empresas
}

\author{
Ricardo M. Pino1, Danny Arévalo-Avecillas ${ }^{2 \star}$, y Carmen Padilla-Lozano \\ (1) CENTRUM Católica Graduate Business School, Pontificia Universidad Católica del Perú, Jr. Daniel Alomía Robles \\ 125, Urb. Los Álamos de Monterrico, Lima, Perú. (correo-e: rpino@pucp.edu.pe). \\ (2) Universidad Católica de Santiago de Guayaquil, Km 1⁄2 vía Carlos Julio Arosemena, Guayaquil, Ecuador. \\ (correo-e: econ.darevalo@gmail.com; carmen.padilla@cu.ucsg.edu.ec).
}

* Autor a quien se debe ser dirigida la correspondencia.

Recibido Abr. 22, 2020; Aceptado Jun. 17, 2020; Versión final Jul. 15, 2020, Publicado Dic. 2020

\begin{abstract}
Resumen
La presente investigación tuvo como propósito validar el efecto positivo del liderazgo servidor en la satisfacción laboral, utilizando como base la teoría del aprendizaje social. Este estilo de liderazgo surgió como un enfoque altruista que prioriza aspiraciones y necesidades de los seguidores. Se administró un cuestionario a una muestra de 225 estudiantes que cursaban la maestría en administración de empresas (MBA, por sus siglas en inglés) en CENTRUM Católica del Perú. El estudio presenta un alcance explicativo, corte transversal y enfoque cuantitativo. El análisis de datos incluyó la aplicación de técnicas como análisis factorial y modelos de ecuaciones estructurales. Los resultados corroboraron la influencia positiva del liderazgo servidor en la satisfacción laboral de los subordinados y demostraron que las dimensiones del liderazgo servidor fueron fielmente replicadas en el contexto peruano. Los hallazgos resaltan la importancia de adoptar enfoques de liderazgo más altruistas en la promoción de la satisfacción laboral, del que deriven mejores actitudes laborales.
\end{abstract}

Palabras clave: satisfacción laboral; subordinados; MBA; medición de liderazgo; liderazgo servidor

\section{Servant leadership and job satisfaction in master of business administration students}

\begin{abstract}
The purpose of this research study was to validate the positive effect of server leadership on job satisfaction based on the social learning theory. The server leadership style emerges as an altruistic approach that prioritizes aspirations and needs of followers. A questionnaire was administered to a sample of 225 students attending the master in business administration (MBA) program at CENTRUM Católica (Peru). The method was explicative, cross-sectional, and quantitative. Data analysis included the application of techniques such as factor analysis and structural equation models. The results corroborated the positive influence of servant leadership on employees' job satisfaction and demonstrated that the dimensions of servant leadership were faithfully replicated in the Peruvian context. These findings highlight the importance of adopting more altruistic leadership approaches in promoting job satisfaction, from which better job attitudes derive.
\end{abstract}

Keywords: job satisfaction; employees; MBA; leadership measurement; servant leadership 


\section{INTRODUCCIÓN}

En entornos operativos inciertos, junto con la presión constante de hacer más con menos, las empresas se han visto en la tarea de redefinir sus roles y responsabilidades tradicionales. Esta necesidad ha obligado a las organizaciones a explorar métodos efectivos para aumentar la satisfacción del cliente interno. En efecto, hoy en día las empresas encuentran complicado el predecir cambios futuros, por lo que la gestión eficiente de los recursos humanos se ha convertido en un componente organizacional central. Diversas compañías reconocen que la gestión de los recursos humanos es la mejor estrategia para obtener ventaja competitiva (Lu et al., 2019). Las organizaciones deben tener la capacidad de descubrir personas competentes, con cualidades de liderazgo, y hacer esfuerzos constantes para promover líderes que mejoren el desempeño de sus subordinados (Haar et al., 2017).

Como miembro de la organización con mayor influencia en el logro de los objetivos empresariales, el líder es el responsable de dirigir la entidad con éxito, de modo que los subordinados puedan desarrollar plenamente sus capacidades. La ejecución de esta misión requiere que el líder aliente a los trabajadores a superar los obstáculos con base en la confianza, presentando una visión clara del futuro de la empresa y estableciendo objetivos específicos. De esta manera, el papel del líder es clave para lograr los objetivos de una organización (Rodríguez-Carvajal et al., 2019). En vista de estos antecedentes, la influencia de los líderes como impulsores en la satisfacción y consiguiente desempeño de sus subordinados ha recibido una creciente atención en la literatura. Inclusive, en las últimas dos décadas, los investigadores han comenzado a examinar los comportamientos específicos de liderazgo exhibidos por los supervisores y cómo influyen en las actitudes y comportamientos de los trabajadores (Alafeshat y Tanova, 2019; Newman et al., 2018).

Las empresas, y la sociedad en general, acogen cada vez más los enfoques de liderazgo que se centran en el bienestar de los seguidores (Van Dierendonck, 2011). Del mismo modo, los seguidores buscan cada vez más líderes que cuiden su relación con ellos, demuestren confiabilidad, desarrollen lealtad en sus equipos y se centren en el crecimiento de los seguidores (Newman et al., 2018). En este sentido, el liderazgo servidor es el mejor ejemplo de este enfoque de liderazgo. Introducido por Greenleaf (1977), y luego desarrollado como una teoría a lo largo del tiempo; el liderazgo servidor hace referencia a líderes cuyo propósito principal es apoyar a sus seguidores a desarrollar todo su potencial en numerosas áreas como la automotivación, el desarrollo de habilidades de liderazgo (Van Dierendonck y Patterson, 2015) y el empoderamiento (Van Dierendonck, 2011). En otras palabras, el líder servidor coloca al colaborador en primer lugar y a él en segundo. Como respuesta a las tendencias actuales y expectativas emergentes, los académicos han recurrido al liderazgo servidor como un modelo que se concentra en el desarrollo del potencial total de los subordinados y tiene la capacidad de enfrentar el desafío de gestionar eficientemente los recursos humanos (Liu, 2019). Al hacerlo, los líderes servidores ayudan a mejorar el capital humano talentoso de los trabajadores. Esto es muy valioso en el actual clima desafiante y altamente competitivo, donde la capacidad de responder y reaccionar a los cambios y las nuevas demandas es primordial para la supervivencia (Ghasabeh, 2018).

El estilo de liderazgo servidor trae beneficios, no solo para el líder, sino para los seguidores también (Mostafa y El-Motalib, 2019). Existen pocas investigaciones sobre cómo los jóvenes ejecutivos perciben otros estilos de liderazgo, más allá de los estilos de liderazgo transaccional y transformacional; esto es cierto, particularmente, en aquellos relacionados con el intercambio de beneficios mutuos para líderes y empleados, como es el caso del estilo de liderazgo servidor (Latif y Marimon, 2019; Muenjohn et al., 2016). Los estudios respecto a liderazgo servidor han incluido organizaciones de bienes y servicios y son claros los beneficios que trae el practicar este estilo de liderazgo; sin embargo, a criterio propio de los autores a partir de sus experiencias personales, los estilos de liderazgo que más se estudian en las escuelas de negocio, centros de formación de líderes por excelencia suelen ser el estilo de liderazgo transformacional y el estilo de liderazgo transaccional y no se suele encontrar, en la malla curricular, temas relacionados con el liderazgo servidor. En las escuelas de negocio se presta atención a la enseñanza de habilidades blandas, como trabajo en equipo, ética, orientación a largo plazo, empatía, etc., relacionándolas con el estilo de liderazgo transformacional. A los estudiantes se les enseña que, tanto la rentabilidad como el clima laboral son necesarios para la sostenibilidad de la empresa y se les transmiten modelos y técnicas para conseguir ambos resultados en las organizaciones. Sin embargo, no se invierte mucho esfuerzo en hacer reflexionar al estudiante respecto a si su rol en la sociedad debería estar marcado más por el servicio a los demás, logrando que los subordinados sean los protagonistas del éxito de las organizaciones, que por conseguir resultados siendo el líder el principal eje del éxito de una organización.

En otras palabras, a los estudiantes de MBA se les enseña a inspirar a sus seguidores a seguirlos, más que a que sean capaces de encontrar su propio camino. Sin embargo, siendo el liderazgo servidor el que logra transformar a los seguidores en líderes servidores también y siendo los graduados de MBA los agentes de cambio en las organizaciones, se hace necesario conocer qué tan positiva es la percepción de los estudiantes de MBA respecto a las características de un líder servidor. Las escuelas de negocios consideran positivo, 
también, enseñar a los estudiantes de MBA cómo tener éxito a través del empoderamiento de los empleados. Sin embargo, no se han encontrado estudios que hayan medido el punto de vista de los estudiantes de MBA con respecto al estilo de liderazgo servidor.

Por otra parte, con base en la teoría del aprendizaje social que enfatiza la importancia del modelado de roles por parte del líder como un proceso central a través del cual se produce la influencia social en el lugar de trabajo (Liden et al., 2014; Van Dierendonck, 2011), es posible pensar que el liderazgo servidor impulse a los seguidores a exhibir niveles más altos de satisfacción. En línea con este enfoque conductual, el presente estudio pretende llenar este vacío, midiendo la relación entre el nivel de identificación de los estudiantes de MBA con el estilo de liderazgo servidor y su satisfacción laboral.

\section{OTROS ANTECEDENTES}

En el contexto de la necesidad de que un líder inicie el cambio, incluso en un entorno cambiante, el liderazgo servidor está recibiendo atención como un nuevo paradigma de liderazgo. En su ensayo "The Institution as Servant", Greenleaf (1977) afirmó que "las personas que desean servir deben, por sí solas, convertirse en constructores de instituciones donde están" (pág. 5). Siguiendo esta definición, los líderes servidores son empresarios institucionales, es decir, agentes de cambio que toman un papel de liderazgo al hacer que los asuntos que valoran sean legítimos y que, también, participan activamente en la implementación del cambio (Rodríguez-Carvajal et al., 2019).

El liderazgo servidor se define como un enfoque holístico y altruista del liderazgo, caracterizado por el enfoque central del líder en las aspiraciones y necesidades de sus seguidores (Van Dierendonck, 2011). En lugar de servir a sus superiores, el líder servidor enfatiza los beneficios para sus seguidores y la comunidad en general. Por ejemplo, un líder servidor escuchará y comprenderá las aspiraciones de sus seguidores y los guiará para lograr estos objetivos. Si bien han comenzado a surgir otros estilos de liderazgo que se centran en los seguidores, el liderazgo servidor sigue siendo único, dado que es fundamental ayudar a los seguidores a alcanzar su máximo potencial y atender las necesidades de los grupos de partes interesadas más amplias (Liu, 2019). Mahatma Gandhi, Madre Teresa de Calcuta y Nelson Mandela son ejemplos destacados de líderes servidores.

En el lugar de trabajo, los líderes son una fuente importante de modelado de roles debido a su estado y poder sobre los seguidores, especialmente cuando son percibidos como un modelo a seguir creíble (Ghasabeh, 2018). En una organización, los gerentes exhiben comportamientos de liderazgo servidor cuando se comprometen con el crecimiento personal y profesional de los empleados por encima de los objetivos financieros de la empresa. De acuerdo con Newman et al. (2018), los líderes influyen significativamente en las actitudes y comportamientos de sus seguidores cuando informan a sus subordinados sobre qué tipos de comportamiento esperan y alinean las recompensas para incentivar dicho comportamiento.

En comparación con otras formas de liderazgo, es más probable que los líderes servidores sean vistos como modelos a seguir creíbles, dado que los seguidores perciben que actúan de manera altruista y están motivados para servir a los demás sin esperar nada a cambio (Brohi et al., 2018). De hecho, en línea con la teoría del aprendizaje social, la emulación de seguidores del comportamiento del líder se ha destacado como una dimensión clave del liderazgo servidor en trabajos anteriores (Van Dierendonck, 2011). A pesar de que el liderazgo transformacional y servidor comparten varias cualidades, el liderazgo servidor es visto como el estilo más sofisticado por la importancia de la humildad y el servicio en la construcción de organizaciones eficaces y el desarrollo de futuros líderes para mantener el crecimiento organizacional (Van Dierendonck, 2011; Vidic et al., 2016).

Liden et al. (2008) evaluaron el liderazgo servidor a través de siete dimensiones: (a) curación emocional, que trata del acto de mostrar sensibilidad a las preocupaciones personales de los demás; (b) creación de valor para la comunidad, que consiste en una preocupación genuina por ayudar a la comunidad; (c) habilidades conceptuales, haciendo referencia a estar efectivamente en la posición de ayudar a otros; (d) empoderamiento, es decir, animar a otros a resolver problemas; (e) apoyo a subordinados a crecer y triunfar, que aborda la preocupación por el crecimiento profesional de los demás; (f) poner a los subordinados primero, que busca satisfacer las necesidades de trabajo de los subordinados como una prioridad; y (g) comportamiento ético, que consiste en interactuar abiertamente, de manera justa y honesta con los demás.

La adaptación rigurosa y el desarrollo del concepto de liderazgo servidor y sus escalas deben establecerse a través de la investigación. En este sentido, los estudios de diferentes países presentaron una evidencia de la existencia del fenómeno del liderazgo servidor y un riguroso proceso de validación de diversas escalas (Hale y Fields, 2007; Zargar et al., 2019). No obstante, más allá de la validación de la existencia del liderazgo servidor como fenómeno, varios estudios han abordado la naturaleza del liderazgo servidor en diferentes 
contextos culturales y su impacto en el entorno laboral, más precisamente en la satisfacción con el trabajo de los subordinados.

La satisfacción laboral es percibida como un estado emocional positivo, producto de la percepción subjetiva de las experiencias de los individuos en el lugar de trabajo (Al-Mahdy et al., 2016). A pesar de que la insatisfacción laboral a veces es causada por circunstancias personales más que por problemas organizacionales, ninguna organización puede ignorar la importancia de la satisfacción laboral de sus subordinados, puesto que representa un factor clave en el éxito organizacional y la productividad. Muchos investigadores han descubierto que la satisfacción laboral es una construcción importante en el bienestar de los empleados, que a su vez es fundamental para el bienestar de la organización (Zargar et al., 2019).

Gran parte de las investigaciones sobre el liderazgo servidor y la satisfacción laboral han sido realizados en el sector servicios, caracterizada por su alto nivel esperado de atención al cliente. Cerit (2009) informó una relación positiva significativa entre el liderazgo servidor y la satisfacción laboral en entornos educativos. Kim y Kim (2017) también confirmaron que el liderazgo servidor y la satisfacción laboral de los subordinados tienen una relación estrecha e influyente, en el contexto de la industria marina. Por su parte, Jang y Kandampully (2018) encontraron que el liderazgo servidor efectivo reduce la intención de renunciar en los subordinados, lo que está relacionado con la mejora de la satisfacción laboral.

Investigadores en diversos campos identificaron que cuanto mayor es la percepción de los empleados sobre el liderazgo servidor en una organización, mayor es su satisfacción laboral (Zargar et al., 2019). De manera similar, Barbuto y Hayden (2011) utilizaron la satisfacción laboral de los empleados como una variable de resultado para la validez predictiva de cada una de las subescalas del liderazgo servidor. Sus resultados indicaron que las sub-escalas auto-administradas de liderazgo servidor se correlacionaron positivamente con la satisfacción de los subordinados. Por lo tanto, se propone la siguiente hipótesis de investigación: $\mathrm{H}_{1}$. Existe un efecto positivo del estilo de liderazgo servidor en la satisfacción laboral de los estudiantes de MBA.

\section{METODOLOGÍA}

El estudio tuvo un enfoque cuantitativo, alcance causal y corte transversal con fuente de datos primarios. El método de recolección de datos fue por medio de cuestionarios que evaluaron el estilo de liderazgo servidor, la satisfacción laboral y otras variables que permitieron la caracterización sociodemográfica de los participantes. Se siguió un método de muestreo por conveniencia, considerando la sugerencia de seleccionar un mínimo de 200 casos para la aplicación de ecuaciones estructurales (Vargas y Mora-Esquivel, 2017).

La unidad de análisis estuvo conformada por estudiantes de MBA a tiempo parcial en CENTRUM Católica, la escuela de negocios más grande del Perú y que cuenta con más de 1,000 estudiantes matriculados por año. Se consideró aquellos maestrantes que eran ejecutivos con al menos dos años de experiencia, mínimo un año laborando para la misma organización y que tenían personal a su cargo en el momento de contestar el cuestionario. Además, los participantes no eran gerentes generales, lo que significaba que tenían un jefe. Se presentó una carta de consentimiento informado a cada maestrante, indicando la finalidad del estudio, estricta confidencialidad sobre los datos y la participación libre y voluntaria. Todos los participantes involucrados asintieron a las condiciones descritas previo a contestar el cuestionario. Siguiendo los criterios de inclusión y exclusión, la muestra final del estudio fue de 225 estudiantes de MBA.

Para el desarrollo de la investigación se distribuyó un cuestionario en forma de encuestas anónimas, con preguntas que evalúan el estilo de liderazgo servidor como variable independiente y la satisfacción laboral como variable dependiente del modelo propuesto. Con respecto al liderazgo servidor, se empleó el cuestionario desarrollado por Liden et al. (2008), que contiene 28 ítems o atributos distribuidos en siete dimensiones que son: (a) curación emocional, (b) creación de valor para la comunidad, (c) habilidades conceptuales, (d) empoderamiento, (e) apoyo a subordinados a crecer y triunfar, (f) poner a los subordinados primero y (g) comportamiento ético. Cada ítem fue evaluado por medio de una escala Likert de siete puntos, donde 1 es totalmente en desacuerdo y 7 es totalmente de acuerdo.

Para la variable dependiente se utilizó la escala de satisfacción laboral de Mohrman-Cooke-Mohrman (MCMJSS por sus siglas en inglés), que incluye ocho ítems relacionados con la satisfacción laboral percibida. Los primeros cuatro ítems miden la satisfacción laboral intrínseca, mientras que los siguientes cuatro ítems miden la satisfacción laboral extrínseca. Los aspectos intrínsecos están relacionados con la autoestima, el crecimiento y desarrollo personal, el logro y las expectativas. Los aspectos extrínsecos están relacionados con el respeto y el trato justo, la información, la cantidad de supervisión por parte del jefe inmediato y la oportunidad de participar en los métodos, procedimientos y objetivos de la organización. El formato de respuesta consistió en una escala Likert de seis puntos, donde 1 es el puntaje más bajo para la satisfacción laboral y 6 el puntaje más alto. 
A pesar de que los instrumentos han sido validados en diferentes estudios, pruebas de confiabilidad fueron ejecutadas para la presente investigación, debido a que los instrumentos se tradujeron al español y se emplearon en una cultura diferente. Se realizó una prueba piloto a 30 estudiantes para verificar que los participantes comprendieran claramente los ítems de ambos cuestionarios y que los datos reflejaran las pretensiones del estudio. Los resultados fueron satisfactorios y entregaron un alfa de Cronbach superior a 0.8 en todos los casos, garantizando la confiabilidad de las escalas.

En primer lugar, el estudio pretendió medir el grado de identificación de los estudiantes de MBA con estilo de liderazgo servidor en su lugar de trabajo. Para ello se emplearon técnicas de estadística descriptiva. Los puntajes promedio de los totales de los ítems relacionados con cada dimensión del liderazgo servidor y los ítems de la satisfacción laboral fueron correlacionados para evaluar el grado de asociación entre las variables del estudio. El estudio pretendió, además, evaluar la relación entre el liderazgo servidor y la satisfacción personal del trabajo. La relación esperada radica en que los ejecutivos que muestran más identificación con el estilo de liderazgo servidor también están más satisfechos en sus trabajos que los ejecutivos que no se identifican con este estilo de liderazgo. Para ello, se realizó un análisis factorial exploratorio con la finalidad de evaluar la estructura interna de ambos modelos y un modelo de ecuaciones estructurales con el propósito de evaluar la relación causal entre ambos constructos. Los datos se tabularon y analizaron con Microsoft Excel, SPSS 24 y el software AMOS.

\section{RESULTADOS}

La Tabla 1 presenta un resumen de variables demográficas de la muestra seleccionada. Se destaca que gran parte de la muestra está representada por participantes del género masculino $(68.89 \%)$, provenientes de Lima (64\%), con experiencia laboral de más de 12 años (40\%), que ocupan cargos de jefatura (88\%) y que tienen entre uno a cinco trabajadores a su cargo (47.35\%).

Tabla 1: Estadística descriptiva de variables demográficas del estudio

\begin{tabular}{|c|c|c|c|}
\hline Variable & Categoría & Cantidad & Proporción \\
\hline \multirow[t]{2}{*}{ Género } & Masculino & 155 & $68.89 \%$ \\
\hline & Femenino & 70 & $31.11 \%$ \\
\hline \multirow[t]{2}{*}{ Lugar de Origen } & Lima & 144 & $64.00 \%$ \\
\hline & $\begin{array}{l}\text { Otras regiones de } \\
\text { Perú }\end{array}$ & 81 & $36.00 \%$ \\
\hline \multirow[t]{3}{*}{ Edad } & 23-30 años & 75 & $33.33 \%$ \\
\hline & 31-40 años & 104 & $46.22 \%$ \\
\hline & Más de 40 años & 46 & $20.44 \%$ \\
\hline \multirow{4}{*}{$\begin{array}{l}\text { Años de experiencia } \\
\text { laboral }\end{array}$} & 2 a 4 años & 40 & $17.78 \%$ \\
\hline & 5 a 8 años & 61 & $27.11 \%$ \\
\hline & 8 a 12 años & 34 & $15.11 \%$ \\
\hline & Más de 12 años & 90 & $40.00 \%$ \\
\hline \multirow{4}{*}{$\begin{array}{l}\text { Años en su empresa } \\
\text { actual }\end{array}$} & 1 a 2 años & 75 & $33.33 \%$ \\
\hline & 3 a 4 años & 46 & $20.44 \%$ \\
\hline & 5 a 6 años & 23 & $10.22 \%$ \\
\hline & Más de 6 años & 81 & $36.00 \%$ \\
\hline \multirow{2}{*}{$\begin{array}{c}\text { Cargo en su empresa } \\
\text { actual }\end{array}$} & Alta dirección & 27 & $12.00 \%$ \\
\hline & Jefatura & 198 & $88.00 \%$ \\
\hline \multirow{4}{*}{$\begin{array}{l}\text { Trabajadores en la } \\
\text { empresa }\end{array}$} & 1 a 49 & 76 & $33.78 \%$ \\
\hline & 50 a 249 & 46 & $20.44 \%$ \\
\hline & 250 a 500 & 11 & $4.89 \%$ \\
\hline & Más de 500 & 92 & $40.89 \%$ \\
\hline \multirow[t]{5}{*}{ Trabajadores a su cargo } & 0 & 0 & $0.00 \%$ \\
\hline & 1 a 5 & 118 & $52.44 \%$ \\
\hline & 6 a 10 & 34 & $15.11 \%$ \\
\hline & 11 a 20 & 31 & $13.78 \%$ \\
\hline & Más de 20 & 42 & $18.67 \%$ \\
\hline \multicolumn{2}{|l|}{$\mathrm{N}$} & 225 & $100.00 \%$ \\
\hline
\end{tabular}

En primera instancia se realizaron pruebas estadísticas para determinar si la estructura de los datos del liderazgo servidor y satisfacción laboral permitía la ejecución de un análisis factorial exploratorio. La medida de adecuación muestral de Kaiser-Meyer-Olkin (KMO) de 0.882 y la prueba de esfericidad de Bartlett con un estadístico chi-cuadrada de $4331.25(p<0.01)$ fueron obtenidas a partir de las respuestas del cuestionario de 
liderazgo servidor. La regla establece que los valores de KMO superiores de 0.7 indican una mejor adecuación de los datos a un modelo factorial; y la hipótesis alternativa de la prueba de esfericidad de Bartlett establece que es posible aplicar un análisis factorial. El cumplimiento de estas condiciones evidenció que la aplicación de un análisis factorial exploratorio es apropiado para la estructura de datos.

En la Tabla 2 se presentan los resultados del análisis factorial exploratorio (AFE) realizado a los 28 ítems del liderazgo servidor. Siete factores presentaron valores propios superiores a la unidad y explican el $66.05 \%$ de la varianza total de los datos. En el análisis individual, los datos replicaron, en distinto orden, las siete dimensiones del liderazgo servidor. De acuerdo con el porcentaje de varianza extraída, estas dimensiones son: (a) creación de valor para la comunidad (11.80\%), (b) habilidades conceptuales $(10.58 \%)$, (c) comportamiento ético (9.64\%), (d) poner a los subordinados primero $(8.92 \%)$, (e) curación emocional (8.90\%), (f) empoderamiento $(8.17 \%)$ y $(\mathrm{g})$ apoyo a subordinados a crecer y triunfar $(8.02 \%)$. Todos los ítems presentaron cargas factoriales mayores que 0.4 , excepto la variable "hace lo que esté a su alcance para hacer más fácil mi trabajo" (0.268), por lo que este ítem fue eliminado del modelo final.

Tabla 2: Cargas factoriales y fiabilidad de las dimensiones del liderazgo servidor

\begin{tabular}{|c|c|c|c|}
\hline Factor/Item & $\begin{array}{l}\text { Cargas } \\
\text { Factoriale } \\
s\end{array}$ & $\begin{array}{l}\text { Varianza } \\
\text { Extraída }\end{array}$ & \begin{tabular}{|l|}
$a d e$ \\
Cronbac \\
$h$ \\
\end{tabular} \\
\hline \multicolumn{2}{|l|}{ Curación Emocional } & $8.90 \%$ & .852 \\
\hline $\begin{array}{l}\text { Me hace sentir que yo podría acudir a él (ella) si tuviera problemas en mi vida } \\
\text { personal. }\end{array}$ & .704 & & \\
\hline Se preocupa por mi bienestar personal. & .777 & & \\
\hline Se da tiempo para hablarme a un nivel personal. & .810 & & \\
\hline Se da cuenta cuando tengo un problema sin siquiera preguntarme. & .513 & & \\
\hline \multicolumn{2}{|l|}{ Creación de Valor para la Comunidad } & $11.80 \%$ & .934 \\
\hline Enfatiza la importancia de contribuir con la comunidad. & .686 & & \\
\hline Está siempre interesado en ayudar a las personas de nuestra comunidad. & .815 & & \\
\hline Está involucrado en actividades de apoyo a la comunidad. & .862 & & \\
\hline Me alienta a participar en actividades de apoyo a la comunidad. & .870 & & \\
\hline \multicolumn{2}{|l|}{ Habilidades Conceptuales } & $10.58 \%$ & .878 \\
\hline Se da cuenta rápidamente cuando algo anda mal. & .624 & & \\
\hline Es capaz de pensar con efectividad cuando hay problemas complejos. & .823 & & \\
\hline Tiene un completo entendimiento de la organización y sus metas. & .735 & & \\
\hline Es capaz de resolver problemas con ideas novedosas. & .690 & & \\
\hline \multicolumn{2}{|l|}{ Empoderamiento } & $8.17 \%$ & .776 \\
\hline Me da la responsabilidad de tomar decisiones importantes en mi trabajo. & .523 & & \\
\hline Me alienta a administrar a mi manera las decisiones importantes de mi trabajo. & .766 & & \\
\hline $\begin{array}{l}\text { Me da libertad para administrar situaciones difíciles de la forma como yo crea que es } \\
\text { mejor. }\end{array}$ & .868 & & \\
\hline Me permite tomar decisiones importantes en mi trabajo sin tener que consultarle. & .402 & & \\
\hline \multicolumn{2}{|l|}{ Apoyo a subordinados a crecer y triunfar } & $8.02 \%$ & .881 \\
\hline Hace de mi desarrollo profesional una prioridad. & .552 & & \\
\hline Se interesa porque logre mis metas en el trabajo. & .709 & & \\
\hline Me provee de experiencias laborales que me permiten desarrollar nuevas habilidades. & .640 & & \\
\hline Se interesa por mis metas de desarrollo de carrera. & .661 & & \\
\hline \multicolumn{2}{|l|}{ Poner a subordinados primero } & $8.92 \%$ & .800 \\
\hline Se interesa más de mi éxito que del suyo propio. & .696 & & \\
\hline Pone mis intereses de desarrollo profesional por encima de los suyos. & .833 & & \\
\hline Sacrifica sus propios intereses para atender los míos. & .869 & & \\
\hline Hace lo que esté a su alcance para hacer más fácil mi trabajo. & .268 & & \\
\hline \multicolumn{2}{|l|}{ Comportamiento ético } & $9.64 \%$ & .830 \\
\hline Mantiene un comportamiento ético excepcional. & .669 & & \\
\hline Es siempre honesto. & .750 & & \\
\hline No comprometería principios éticos para alcanzar el éxito profesional. & .613 & & \\
\hline Valora la honestidad por encima de las ganancias monetarias. & .705 & & \\
\hline \multicolumn{2}{|l|}{ Modelo Completo } & $66.05 \%$ & .923 \\
\hline
\end{tabular}

El coeficiente alfa de Cronbach permitió evaluar la confiabilidad de los instrumentos empleados, precisamente debido a que éstos pasaron por un proceso de traducción y adaptación al contexto peruano. Se calculó una estimación de consistencia interna para el instrumento de liderazgo servidor y el valor alfa del modelo general 
fue de 0.923 . Además, el valor alfa de las siete dimensiones fue superior al umbral de 0.70 sugerido por la literatura.

En la Tabla 3 se reportan los resultados del análisis factorial exploratorio (AFE) realizado a los 8 ítems de la satisfacción laboral. Previamente las pruebas estadísticas de adecuación muestral KMO de 0.920 y la prueba de esfericidad de Bartlett con un estadístico chi-cuadrada de $870.54(\mathrm{p}<0.01)$ fueron satisfactorias, indicando que es posible aplicar un análisis factorial para la estructura de datos. De acuerdo con esta técnica, un solo factor presentó un valor propio superior a la unidad y explica el $52.06 \%$ de la varianza total de los datos. Bajo este escenario, no se observaron diferencias entre los ítems que explican factores intrínsecos y extrínsecos de la variable dependiente, por lo que el factor resultante se denominó satisfacción laboral. En el análisis individual, todos los ítems presentaron cargas factoriales mayores que 0.4, excepto el grado de satisfacción sobre la cantidad de supervisión que recibe en el lugar de trabajo (0.264), por lo que este ítem fue eliminado del modelo final. El valor alfa de la satisfacción laboral fue de 0.877 , por encima del umbral sugerido por la literatura, indicando que el instrumento presenta una adecuada consistencia interna

Tabla 3: Cargas factoriales y fiabilidad de la dimensión de la satisfacción laboral

\begin{tabular}{|l|l|l|l|}
\hline Factor / Item & Cargas Factoriales & $\begin{array}{l}\text { Varianza } \\
\text { Extraída }\end{array}$ & $\begin{array}{l}\alpha \text { de } \\
\text { Cronbach }\end{array}$ \\
\hline Satisfacción laboral & $52.06 \%$ & .877 \\
\hline $\begin{array}{l}\text { El sentimiento de autoestima o respeto a sí mismo que obtiene por } \\
\text { estar en su trabajo }\end{array}$ & .755 & & \\
\hline La oportunidad que tiene crecimiento personal en su trabajo & .777 & .794 & \\
\hline El sentimiento de tener logros significativos en su trabajo & .717 & & \\
\hline $\begin{array}{l}\text { Su trabajo actual al considerar las expectativas que tenía de éste } \\
\text { cuando tomó el empleo }\end{array}$ & & \\
\hline El respeto y trato justo que recibe de sus superiores & .794 & & \\
\hline El sentimiento de estar informado sobre su trabajo & .798 & & \\
\hline La cantidad de supervisión que recibe & .264 & & \\
\hline $\begin{array}{l}\text { La oportunidad para participar en la determinación de métodos. } \\
\text { procedimientos y metas }\end{array}$ & .715 & & \\
\hline
\end{tabular}

\section{Relación entre Liderazgo Servidor y Satisfacción Laboral}

Los valores de cada dimensión del liderazgo servidor y el valor de la satisfacción laboral fueron obtenidos a través del promedio de las puntuaciones totales de los ítems que compone cada dimensión. Los resultados de estadística descriptiva derivados del instrumento de liderazgo servidor destacaron que poner a subordinados primero fue la dimensión con la puntuación más baja $(\bar{x}=4.84)$, seguido de la dimensión de curación emocional $(\bar{x}=5.01)$. Por otro lado, las habilidades conceptuales $(\bar{x}=6.29)$ y comportamiento ético $(\bar{x}=6.49)$ fueron las dimensiones con las puntuaciones más altas.

Tabla 4: Estadística descriptiva y correlaciones entre variables latentes del liderazgo servidor y satisfacción laboral

\begin{tabular}{|c|c|c|c|c|c|c|c|c|c|c|}
\hline Variable Latente & Media & D.E. & 1 & 2 & 3 & 4 & 5 & 6 & 7 & 8 \\
\hline \multicolumn{11}{|l|}{ Liderazgo servidor } \\
\hline 1. Curación Emocional & 5.01 & 1.28 & 1 & & & & & & & \\
\hline 2. Creación de Valor para la Comunidad & 5.15 & 1.27 & $.572^{\star * *}$ & 1 & & & & & & \\
\hline 3. Habilidades Conceptuales & 6.29 & 0.81 & $.362^{* * *}$ & $.431^{* \star *}$ & 1 & & & & & \\
\hline 4. Empoderamiento & 5.95 & 0.78 & $.289^{\star * \star}$ & $.340^{\star * *}$ & $.405^{\star \star *}$ & 1 & & & & \\
\hline 5. Apoyo a subordinados a crecer y triunfar & 6.10 & 0.80 & $.378^{* * *}$ & $.421^{* * *}$ & $.556^{* * *}$ & $.530^{* * *}$ & 1 & & & \\
\hline 6. Poner a subordinados primero & 4.84 & 1.11 & $.367^{\star \star \star}$ & $.362^{\star * \star}$ & $.223^{\star \star *}$ & $.428^{* * *}$ & $.474^{\star \star \star}$ & 1 & & \\
\hline 7. Comportamiento ético & 6.49 & 0.72 & $.268^{* * *}$ & $.309^{\star * *}$ & $.532^{\star * \star}$ & $.340^{* * *}$ & $.579^{\star \star \star}$ & $.290^{* * *}$ & 1 & \\
\hline 8. Satisfacción Laboral General & 4.59 & 0.80 & $.275^{\star \star \star}$ & $.203^{\star * *}$ & $.154^{* *}$ & $.161^{* *}$ & $.240^{* \star *}$ & $.151^{* *}$ & $0.120^{*}$ & 1 \\
\hline
\end{tabular}

En la Tabla 4 se reporta el grado de asociación entre las dimensiones del liderazgo servidor y la satisfacción laboral por medio de los coeficientes de correlación de Pearson. Los coeficientes significativos a un nivel de confianza de $99 \%, 95 \%$ y $90 \%$ fueron marcados con $\left({ }^{* *}\right),\left({ }^{* *}\right)$ y $\left({ }^{*}\right)$, respectivamente. Se destaca que las dimensiones de curación emocional $(r=0.248, p<0.01)$, creación de valor para la comunidad $(r=0.203$, $p<0.01)$ y apoyo a subordinados a crecer y triunfar $(r=0.216, p<0.01)$ presentaron asociaciones positivas fuertes con respecto a la satisfacción laboral. Además, se aprecia una asociación positiva entre las siete dimensiones, lo que sugiere que pueden ser explicadas por un factor de segundo orden, liderazgo servidor. Por otra parte, la dimensión de comportamiento ético presentó un coeficiente significativo al $90 \%$ de nivel de confianza en la relación con la satisfacción laboral. Las tres dimensiones restantes, habilidades conceptuales, 
empoderamiento y poner a subordinados primero también presentaron coeficientes significativos, pero al $95 \%$ de nivel de confianza.

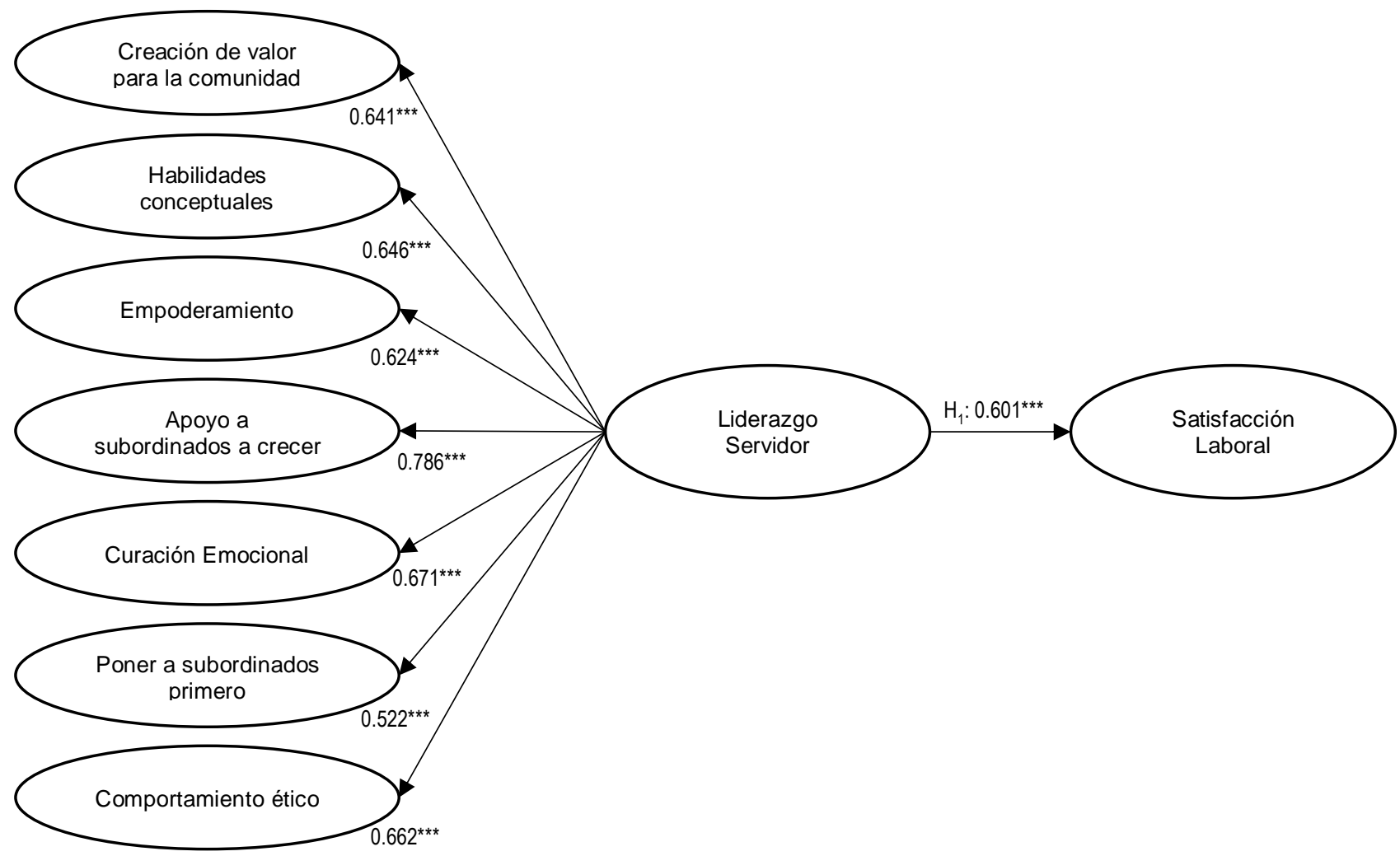

Figura 1: Resultados del modelo de investigación

A partir de estos resultados, el siguiente paso consistió en realizar la evaluación del modelo estructural. El modelo y la hipótesis de investigación propuesta fueron comprobadas utilizando la técnica de modelos de ecuaciones estructurales (Figura 1). La Tabla 5 presenta todas las relaciones del modelo, los coeficientes estandarizados, la razón crítica o valor $z$ y la significancia de cada relación. Los tres asteriscos de cada ruta representan un coeficiente significativo a un nivel de confianza de $99 \%$. En primera instancia, los resultados evidencian que todas las rutas estimadas entre las siete dimensiones del liderazgo servidor y el constructo de segundo orden fueron significativas, lo que sugiere validez convergente. El efecto directo entre el constructo de segundo orden del liderazgo servidor y el constructo de satisfacción laboral fue de 0.342 , significativo a un nivel de confianza del 99\% $(\mathrm{p}<0.001)$. Por esta razón, la hipótesis de investigación $\left(\mathrm{H}_{1}\right)$ fue aceptada, demostrando que el liderazgo servidor influye positivamente en la satisfacción laboral de los subordinados. Después de eliminar los ítems con cargas factoriales débiles y refinar el modelo, los resultados obtenidos como el estadístico de chi-cuadrada normado $\left(\mathrm{X}^{2} / d f\right)=1.916$, el índice de ajuste comparativo $(\mathrm{CFI})=0.90$ y el error cuadrático medio de aproximación $(\mathrm{RMSEA})=0.064$ demuestran que, en efecto, la bondad de ajuste del modelo completo es aceptable para la muestra considerada.

Tabla 5: Resultados de las relaciones del modelo de ecuaciones estructurales

\begin{tabular}{|l|l|l|l|l|l|}
\hline Relaciones & Coeficiente & S.E. & $\begin{array}{l}\text { Coeficiente } \\
\text { Estandarizado }\end{array}$ & C.R. & P < \\
\hline Creación de Valor para la Comunidad <-- Liderazgo servidor & 1 & & 0.585 & & .001 \\
\hline Habilidades Conceptuales <-- Liderazgo servidor & 0.949 & 0.148 & 0.715 & 6.409 & .001 \\
\hline Empoderamiento <-- Liderazgo servidor & 0.61 & 0.106 & 0.638 & 5.73 & .001 \\
\hline $\begin{array}{l}\text { Apoyo a subordinados a crecer y triunfar <-- Liderazgo } \\
\text { servidor }\end{array}$ & 1.122 & 0.162 & 0.857 & 6.933 & .001 \\
\hline Curación Emocional <-- Liderazgo servidor & & & & & \\
\hline Poner a subordinados primero <-- Liderazgo servidor & 0.384 & 0.102 & 0.499 & 3.747 & .001 \\
\hline Comportamiento ético <-- Liderazgo servidor & 0.707 & 0.115 & 0.695 & 6.127 & .001 \\
\hline Satisfacción Laboral General <-- Liderazgo servidor & 0.342 & 0.093 & 0.309 & 3.685 & .001 \\
\hline
\end{tabular}


El propósito de este estudio fue validar el efecto positivo del liderazgo servidor en la satisfacción laboral intrínseca y extrínseca de los subordinados, analizando cada dimensión del liderazgo servidor en esta muestra. Los resultados mostraron que las siete dimensiones establecidas por Liden et al. (2008) fueron fielmente replicadas en la muestra actual con apropiada fiabilidad y validez discriminante. Además, los resultados entregan una mayor confianza en el uso de este instrumento en el contexto peruano y que comparte rasgos de liderazgo similares a otros países de la región latinoamericana.

Los hallazgos de la investigación son consistentes con otros estudios que han encontrado evidencia de validez factorial de las medidas del liderazgo servidor utilizando el instrumento de Liden et al. (2008) en diversos contextos, como en Europa (Bobbio et al., 2012) y China (Schaubroeck et al., 2011); y con resultados similares en contextos como en Estados Unidos (Hale y Fields, 2007). Sin embargo, otros estudios han identificado otras dimensiones (Sendjaya et al., 2019) o empleado versiones cortas del instrumento original (Liden et al., 2015), con resultados satisfactorios (Lu et al., 2019), sugiriendo que podría haber otros factores además de los contextos culturales que afectan la interpretación del liderazgo servidor. Cabe destacar, además, que los resultados del estudio también sugieren que el liderazgo servidor puede ser representado como un constructo de segundo orden, inclusive cuando se evalúa con todas sus siete dimensiones (Liden et al., 2015). Una modelación multidimensional en un constructo de segundo orden asegura que el instrumento captura integralmente varios detalles del liderazgo servidor y facilita la prueba de hipótesis relacionadas con los antecedentes o impactos del liderazgo servidor de una manera más simple y eficiente.

Al probar la estructura de segundo orden del liderazgo servidor y su impacto en la satisfacción laboral de los empleados, este estudio demuestra la naturaleza holística del liderazgo servidor. Los resultados del modelo estructural también son consistentes con los hallazgos anteriores en otros contextos (Barbuto y Hayden, 2011), principalmente en el campo de la educación, que relacionan el liderazgo servidor con la satisfacción laboral (Al-Mahdy et al., 2016; Cerit, 2009). Además, proporcionan mayor evidencia al impacto que el liderazgo servidor tiene tanto en la satisfacción laboral extrínseca, que se relaciona con la dimensión de higiene, cuyos componentes incluyen la supervisión y las relaciones interpersonales, como en la satisfacción laboral intrínseca de los subordinados, que se relaciona con aspectos que ayudan a encontrar más significado interno en el lugar de trabajo. La investigación ayuda a resaltar la importancia de adoptar enfoques de liderazgo más altruistas como el liderazgo servidor en la promoción de la satisfacción laboral de los empleados. Los líderes en la región deben ser conscientes de los resultados positivos de este enfoque que, eventualmente, puede contribuir al compromiso de los subordinados y mejorar su desempeño que son aspectos esenciales para las organizaciones contemporáneas (Sony y Mekoth, 2016).

A pesar de que tradicionalmente, la satisfacción laboral se ve afectada por aspectos relacionados con salarios, relaciones con los colegas, el tipo de trabajo, el progreso profesional y otros incentivos organizacionales, la investigación demostró que el liderazgo servidor se asocia significativamente con la satisfacción laboral, de la cual derivan mejores actitudes laborales, menor rotación, mejor comportamiento de ciudadanía organizacional (OCB), creatividad y mayor rendimiento (Feng et al., 2015; Van Dierendonck, 2011). No obstante, el coeficiente sugiere que otros factores pudieran incrementar la explicación de la satisfacción laboral.

Las implicancias de la presente investigación son, por el lado gerencial, que desarrollar un estilo de liderazgo servidor es valioso para los líderes empresariales, ya que al hacerlo crean valor para la comunidad, mejoran las habilidades de sus subordinados, les permiten desarrollarse y ampliar sus horizontes. Por el lado del colaborador, el mejorar la satisfacción en el trabajo está relacionado con una menor rotación y mayor identificación con la empresa. Por el lado de la academia, la contribución de esta investigación ha sido mostrar que los estudiantes de maestría valoran en sus superiores el estilo de liderazgo servidor, por lo que las escuelas de negocio podrían alinear la malla curricular de sus programas para que contribuyan con el desarrollo de estas características de liderazgo en sus estudiantes. De acuerdo a los resultados del estudio, las escuelas de negocio harían bien en dedicar más horas a la enseñanza de los beneficios de empoderar a los colaboradores, ayudarlos a utilizar sus capacidades al límite y a desarrollar nuevas, así como a mantener un comportamiento ético en todo momento.

Se destacan varias limitaciones a considerar en la interpretación de los resultados. En primer lugar, el liderazgo servidor se midió a través de las percepciones de los subordinados con respecto a los diversos aspectos de las orientaciones de liderazgo del supervisor inmediato lo que dificulta la construcción de una medida objetiva, a pesar de que esta resulta una práctica común en estudios de liderazgo. En segundo lugar, los datos transversales limitan la capacidad de demostrar la causalidad de que el liderazgo servidor conduce a la satisfacción de los empleados. Por último, la muestra se seleccionó en función de la conveniencia y facilidad de acceso y representaba a estudiantes de maestría que laboran en empresas de múltiples sectores económicos y, por tanto, mitiga la réplica de estos hallazgos. 


\section{CONCLUSIONES}

Las siguientes conclusiones surgen a partir de los hallazgos de la presente investigación: 1. los resultados permitieron corroborar el efecto positivo del liderazgo servidor en la satisfacción laboral de los subordinados, con estimadores satisfactorios en la aplicación de ecuaciones estructurales y validación de instrumentos; 2. El estudio demostró que las siete dimensiones del instrumento original de liderazgo servidor fueron fielmente replicadas con fiabilidad y validez discriminante satisfactorias; 3 . El análisis realizado sugirió que el liderazgo servidor puede ser representado como un constructo de segundo orden y facilita la prueba de hipótesis relacionadas con los antecedentes o impactos del liderazgo servidor de una manera más simple y eficiente; 4. Los hallazgos resaltan la importancia de adoptar enfoques de liderazgo más altruistas como el liderazgo servidor en la promoción de la satisfacción laboral; es decir que personas de niveles jerárquicos superiores acojan conductas y rasgos inherentes al liderazgo servidor del cual deriven mejores actitudes laborales, menor rotación, mejor comportamiento, creatividad y mayor rendimiento por parte de los subordinados.

\section{REFERENCIAS}

Alafeshat, R. y Tanova, C., Servant Leadership Style and High-Performance Work System Practices: Pathway to a Sustainable Jordanian Airline Industry, Sustainability, ISSN: 2071-1050, 11(6191), 1-21 (2019)

Al-Mahdy, Y., Al-Harthi, A. y Salah. N., Perceptions of School Principals' Servant Leadership and their Teachers' Job Satisfaction in Oman, Leadership and Policy in Schools, ISSN: 1570-0763, 15(4), 543-566 (2016)

Barbuto, J. y Hayden, R., Testing Relationships between Servant Leadership Dimensions and Leader Member Exchange, Journal of Leadership Education, ISSN: 1552-9045,10(2), 22-37 (2011)

Bobbio, A., Dierendonck, D.V. y Manganelli, A.M., Servant Leadership in Italy and Its Relation to Organizational Variables, Leadership, ISSN: 1742-7150, 8(3), 229-243 (2012)

Brohi, N.A., Jantan, A.H. y otros cuatro autores, The Impact of Servant Leadership on Employees Attitudinal and Behavioural Outcomes, https://doi.org/10.1080/23311975.2018.1542652, Cogent Business and Management, ISSN: 2331-1975, 5, 1-17 (2018)

Cerit, Y., The Effects of Servant Leadership Behaviours of School Principals on Teachers' Job Satisfaction, Educational Management Administration \& Leadership, ISSN: 1741-1432, 37(5), 600-623 (2009)

Feng, X., Verdorfer, A.P. y Peus, C.V., Thriving in Turbulent Times: Servant Leadership as a Pathway to Employee Wellbeing and Retention, https://doi.org/10.5465/ambpp.2015.17726abstract, Proceedings (2015)

Ghasabeh, M. Understanding Why Servant Leadership Works For Employee Engagement, Leadership Excellence, 35(8), 56-57 (2018)

Greenleaf, R. K., Servant Leadership: A Journey into the Nature of Legitimate Power and Greatness, Paulist Press, New York, USA (1977)

Haar, J., Brougham, D., y otros autores, Servant Leadership and Work Engagement: The Mediating Role of Work-Life Balance. New Zealand Journal of Human Resources Management, ISSN: 11755407, 17(2), 56-72 (2017)

Hale, J.R. y Fields, D.L., Exploring Servant Leadership Across Cultures: A Study of Followers in Ghana and The USA, Leadership, ISSN: 1742-7150, 3(4), 397-417 (2007)

Jang, J. y Kandampully, J., Reducing Employee Turnover Intention through Servant Leadership in the Restaurant Context: A Mediation Study of Affective Organizational Commitment, International Journal of Hospitality \& Tourism Administration, ISSN: 1525-6480, 19(2), 125-141 (2018)

Kim, J.H. y Kim, M., The Influence of Captains' Servant Leadership on Leader Trust, Job Satisfaction, and Job Performance in a Marine Industry, International Journal of Economic Perspectives, ISSN: 1307-1637, 11(2), $490-499$ (2017)

Latif, K. F., y Marimon, F., Development and validation of servant leadership scale in Spanish higher education, https://doi.org/10.1108/LODJ-01-2019-0041, Leadership \& Organization Development Journal, 40(4), $499-519$ (2019)

Liden, R.C., Wayne, S.J. y otros dos autores, Servant Leadership: Development of a Multidimensional Measure and Multilevel Assessment, The Leadership Quarterly, ISSN: 1048-9843, 19(2), 161-177 (2008)

Liden, R.C., Wayne, S.J. y otros dos autores, Servant Leadership: Validation of a Short Form of the SL-28, The Leadership Quarterly, ISSN: 1048-9843, 26(2), 254-269 (2015)

Liu, H., Just the Servant: An Intersectional Critique of Servant Leadership, Journal of Business Ethics, ISSN: 0167-4544, 156, 1099-1112 (2019)

Lu, J., Zang, Z. y Jia, M., Does Servant Leadership Affect Employees' Emotional Labor? A Social Information Processing Perspective, Journal of Business Ethics, ISSN: 0167-4544, 159, 507-518 (2019)

Mostafa, A.M. y El-Motalib, E.A., Servant Leadership, Leader-Member Exchange and Proactive Behavior in the Public Sector, https://doi.org/10.1177/0091026018816340, Public Personnel Management, ISSN: 0091-0260, 48(3), 309-324 (2019) 
Muenjohn, N., Pimpa, N. y otros dos autores, Developing leadership curriculum for business education program in Asia, The Journal of Developing Areas, 50(5), 443-451 (2016)

Newman, A., Neesham, C. y otros dos autores, Examining the influence of servant and entrepreneurial leadership on the work outcomes of employees in social enterprises, https://doi.org/10.1080/09585192.2017.1359792, International Journal of Human Resource Management, 29(29), 2905-2926 (2018)

Rodríguez-Carvajal, R., Herrero, M. y otros tres autores, Servant Leadership and Goal Attainment through Meaningful Life and Vitality: A Diary Study, Journal of Hapiness Studies, ISSN: 1389-4978, 20, 499-521 (2019)

Schaubroeck, J., Lam, S.S. y Peng, A.C. Cognition-Based and Affect-Based Trust as Mediators of Leader Behavior Influences on Team Performance, Journal of Applied Psychology, ISSN: 0021-9010, 96(4), 863-871 (2011)

Sendjaya, S., Eva, N. y otros tres autores, SLBS-6: Validation of a Short Form of the Servant Leadership Behavior Scale, Journal of Business Ethics, ISSN: 0167-4544, 156, 941-956 (2019)

Sony, M., y Mekoth, N., The Relationship between Emotional Intelligence, Frontline Employee Adaptability, Job Satisfaction and Job Performance, Journal of Retailing and Consumer Services, ISSN: 0969-6989, 30, 20-32 (2016)

Van Dierendonck, D., Servant Leadership: A Review and Synthesis, Journal of Management, ISSN: 0149-2063, 37(4), 1228-1261 (2011)

Van Dierendonck, D., y Patterson, K., Compassionate Love as a Cornerstone of Servant Leadership: An Integration of Previous Theorizing and Research, Journal of Business Ethics, ISSN: 0167-4544, 128(1), 119-131 (2015)

Vargas, T., y Mora-Esquivel, R., Tamaño de la muestra en modelos de ecuaciones estructurales con constructos latentes: Un método práctico, http://dx.doi.org/10.15517/aie.v17i1.27294, Actualidades Investigativas en Educación, 17(1), 1-34 (2017)

Vidic, Z., Burton, D. y otros tres autores, Emotional and motivational correlates of leadership styles: A comprehensive framework for understandiing effective leaders, http://dx.doi.org/10.1002/jls.21485, Journal of Leadership Studies, 10(3), 22-40 (2016)

Zargar, P., Sousan, A. y Farmanesh, P., Does Trust in Leader Mediate the Servant Leadership Style-Job Satisfaction Relationship? Management Science Letters, ISSN: 1923-9343, 9, 2253-2268 (2019) 
